Monatsschrift für Geburtshülfe und Gynaekologie 1895;1:200-202

\title{
Tagesnachricliten und Notizen
}

Weihiiachtstage in Paris. 1894. - A. Martin. Ein viertägiger Auf-enthalt in Paris zwischen Weihnachten und Neujahr 1894 gab mir die $\Lambda$ villkommene Gelegenheit, mich mit einem grossen Teil unserer Pariser Fachgenossen in Ver-bindung zu setzen. Ich kann nicht genug die kollegiale Liebenswürdigkeit rühmen. mit der ich von alien Soiten auf'genommen worden bin. Trotz der ungünstigen Jahreszeit, in der aucli in Paris jeder es gern vermeidet, ein Krankenhaus aufzusuehen, wurde mir die erwünschte G-elegenheit geboten, eine Ft'ille interessanter Fälle zu selien und die neuen Hospitaleinriehtungen kennen zu lernen. darch welclie sich die Fortentwickelung auf diesem, in Paris auffallend lango stagnierenden, Gebiete erkennbar macht. - Für gynäkologische, besser gesagt, operative Kranke. hat Póan im „Hôpital international” ein geradezu mustergültiges Krankenhaus geschaft'en. Lange Jahrzehnte hatte er im Hôpital St. Louis sich vergeblich um gründliche Ret'ormen bemiibt. Als er an der Altersgrenze angelangt seinen dortigen Posten aufgeben musste, hat er hier, Rue de Santé, auf einem von der Stadt ihm bewilligten Terrain eine wahre Musteranstalt ge.scbaffen. Sie dient nicht ausschliesslich den Frauenkrankbeiten noch auch der gynäkologisclien Ghirur-gie, doch ist dieser unzweifelhaft der bevorzugte $\Gamma$ latz eingeräumt. In dem nach der Strasse zu liegende $\pi$ Hauptbau beñnden sich auch noch mehrere nicht chirur-gische Polikliniken. Das ganze Haus mit seiner streng durchgefübrten $\mathrm{Re}^{1 / 8} / \mathrm{li}<\cdot 1 \mathrm{l}-\mathrm{keit}$, seinen Vorkehrungen für Desinfectton, der vortreffüchen Ventilation und Centralheizung, den Einrichtungen zur Erzeugung des elektrischen Lichtes, ist schon mehrere Male für anderweitige Hospitaler vorbildlich geworden.

Untor Pinard's Leitung hat sich im „Hôpital Baudelocque” eine ausgedehnte Barackenanlage für geburtshülfiiche Zwecke entwickelt, die in ihrer Art ebenfalls als mustergültig genannt werden darf. Zweistöckige Wöchnerin-Baracken, mit isoliert stehenden Krankenbaracken, Museum und Ijaboratorium, nehmen für die bestimmte Frist von einigen Monaten eine beschränkte Zahl von Studenten auf, welclie alsdann ausschliesslich mit Geburtshülfe und G-ynäkologie beschäftigt werden. Varnier steht als Agrégé Pinard zur Seite. Alle grösseren gynäkologisclien Operationen vollzioht als Chirurg Segond im Hospital selbst.

Ein grosser Neubau ist tiir das „Hôpital Broca” - früher Lourcine im Werden: Pozzi bewältigt das reiche Material dieses Hauses als gynäkologi-scher Chirurg zunächst in den wenig schönen alten Räumen.

Einer der Zwecke meines Besuches $\Lambda$ var, die Morcellement-Operation an ihrer Entwíckelungsstätte kennen zu lernen. Ich kann nur mit Bewunderung davon berichten, wie Péan und sein Schüler Segond zum Teil sehr schwierige Fälle von Myomen auf diese Weise per vaginam entwickelten. Die Operation macht in ihrer Hand den Eíndruck vollkommener Sicherheit und die Verwendung der Pinces erscheint so wenig schwierig, dass jeder Zweifel an der Zweckdienlich-keit derselben schwinden muss. In keinem der 5 Fälle, welche ich von Péan's Meisterhand operieren sah, ebenso wenig wie in dem von Segond operierten, kam es zu einer auch nur unbequemen Blutung. Ich habe bei Beiden, ebenso wie auf Pozzi's Station, eine grosse Anzahl von Reconvalescenten in alien Stadien p.-operat. gesehen, welche durchaus den Eindruck einer ungestörten Genesung machten. 
Tagesnacbrichten und Notïzen.

201

Einen Fall von Hysterectomie bei Beckeneiterung habe ich leider in diesen Tagen nicht zu sehen bekomraen: und gerade darauf hatte ich grosses Gewicht gelegt, denn in den Myomfällen schien mir díeser Weg keine wesentlichen Vor-teile vor den bei uns üblichen zu bieten: vaginale Operation niit Ligatiiren bei geringem, Operation nach Koeliotoraie bei grösserem Ümfang. Uebrigens hat die vaginale Hysterectomie mit Morcellement und Pinces in den verschiedenen Modifikationen des ursprünglichen Pé an'sehen Verfahrens sich unzweifelhaft nahezu die AUeinherrschaft bei den Parisern errungen. Ganz be-sonders schienen die Kranken selbst diese „neue” Methode vor der Koeliotomie zu bevorzugen. Unverkennbar sind die Genesungsresultate ganz übe $\pi v i e g e n d$ günstige, wobei der Anteil einer strong durchgeführten Anti- und Aseptik nicht untersehätzt werden soil.

Meinen Zweifeln bezüglich der Durchführbarkeit des Verfahrens bei sehr ausgedehnten Verwachsungen wurde von den verschiedenen Kollegen entgegen-gehalten, dass es in solchen verzweifelten Fallen nicht darauf ankomme, wirklich alle Eiterherde vollständig zu beseitigon. Es gonüge, sie weit zu eröfi $3 / 4$ en und nach unten drainiert zu erhalten: nach wenigen Wochen erfolge fast aus-nahmslos Schrumpfung und Heilung, selbst da, wo Nachbarorgane in den Erkran-kungsherd hineingezogen und auch im Vorlauf der Operation oder der Aus-heilung eröffnet wurden. In demselben Sinne hat bekanntlich auch L. Landau sich ausgesprochen, welcher diese Operation in Berlin in ausgedehntem Masse übt. Für mích liegt die mir noch zunächst unüberwindliche Schwierigkeit in der Gefahr diagnostischer Irrtümer und in der Schwierigkeit oxacter Kontrolle des Operationsfeldes. Diese lässt sich bei den Fallen relativ bequemer Zugäuglich-keit des Uterus - - davon habe ich mích überzeugt - gewiss durchführen: ob abcr in den schwierigen, mit allseitigen Yerwachsungen und eitriger Schmelzung dor unter einander zu einem kaum entwirrbaron Ganzen verschmolzenen Becken-eingeweide - davon habe ich leider keine eigne Anschauung erhalten. - Hoti'ent-lich bringt sie mir ein späterer Besuch zu günstigerer Jahreszeit!

In No. 50 der „Münchener Medicin. Wochenschrift” 1894 ist unter dem Titel „Katholische KircL·e und niediciiiische YVissenschaft" ein Vorkommnis mitgeteilt. welches auch in politischen Kreisen Aufmerksamkeit erregte, aber doch mehr den ärztlichen Stand, besonders den praktischen Geburtshelfer angeht.

Bei einer Gravida im 3. Monate, leitete der praktische Arzt Dr. H. in D., auf Grand von Indicatio vitalis durch Einlegung - eines Bougie den Abortus ein. Am Nachmittage desselben Tages besuchte die Ivranke der Pfarrer 11. Als er die Massnahmen des Arztes erfuhr, brachte er die Frau durch die furchtbarsten Drohungen von zeitlicher und e $\lambda$ viger Verdammnis dahin, dass sie das Bougie selbst wieder herauszog. Er soil sogar zu dem Ehemann gesagt haben, ,er köime den Doktor wegen Kindesabtreibung in das Zuchthaus bringen," eine Aeusserung, $\Lambda$ velche allerdings später von ihm in Abrede gestellt wurde. Drei Tage später gebar die ohnehin ki > rperlich sehr heruntergekommene und in die hochgradigste psychische Erregung versetzte Frau eine macerirte Frucht aus dem 3. Monat.

Auf die von dem Arzte gegen den Pfarrer bei dem bischöflichen Ordinariate in Augsburg erhobene Beschwerde, bekam der Arzt keine Genugthuung, sondern das $\Lambda$ rerhalten des Pfarrers wurde als ein durchaus korrektes bezeichnet, indem sich die geistliche Behörde auf Aussprüche von Vertretern der katholischen Moral-theologie und Pastoral-Medicin, wie den heíligen Alphons Liguori, Pruner, Dr. Alfers und Dr. Kappelmann berief. 
Die „Münch. medic. Wochenschr.” äussert in ihrer den ärztlichen Stand-punkt energisch wahrenden Nachschrift, die Zuvorsicht, dass damit von bischöf-licher Seite noch nicht das letzte Wort gesprochen sei.

Es versteht sich wohl von selbst, dass der in einer „katholischen Gegend” thätige Geburtshelfer, gleichviel welcher Confession er selbst a agehört und welchen religiösen Anschauungen er huldigt, in Ausiibung seiner Thätigkeit auf die reli-giösen Empfindungen seiner Klienten so $\Lambda$ vohl wie auch auf die Forderungen der Pastoral-Medicin die weitgehendste Ilücksicht zu nehmen hat, docb unbeschadet anerkannter Lehren der medicinischen Wissenschaft. Gerade auf dem Gebiete der Geburtshülfe sind in dem Widerstreit von Theologie und Medicin schon oft Konfiikte vorgekommen, $\Lambda$ vobei die Medicin stets den, freilich nur auf das leibliche Wohl abzielenden, humaneren Standpunkt vertrat.

202 Tagesnaehrichten und Notizen. - Personal-Nachrichten.

Es ist allerdings sehr zu beklagen, dass das bíschöfliche Ordinariat zu Augsburg gerade in Anknüpi'ung an das E1/8zelvorkommnis und die dabei beteilig-ten Personen, sich nicht zu einem liölieren Gesich·t spunk te aufgescliwungen und vielmehr Schritte in Aussicht gestellt hat, welche sämtliche veraltete Satzungen der Pastoral -Medicin mit den so ausserordentlich umgestaltcten Lehren der modern en Geburtshülfe in Einklang zu bringen vermöchten. Es mag zugegeben werden, dass ein derartiges Unternehmen innerhalb der hierarchischen Kreise, von welchen ein soldier Umschwung ausgelien müsste, aut' g·rosse Schwierigkeiten stösst, weshalb aber gerade zum Mindesten ein „tolerari posse” geiibt werden sollte.

Dass die katholische Kirche sehr wohl aueh den Fortschritten der medici-nischen Wissenschaft $\mathrm{zu}$ folgen und - wenigstens in jtalien - ihren Forderungen stattzugeben vermag, bewe $1 / 8 \mathrm{t}$ die berühmte von Porro angerufene Entscheidung des Bischofs von Pavia, welche dadurch flir die Ausbreitung der nach ihm ge-nannten Operation von grosser ßedeutung wurde.

Für den II. internationaleii gynäkologischen Congiess, welcher im September 1895 in Genf abgelialten werden soil, sind folgende Themata in Aussicht genommen:

Behandlung der Eklampsie.

Operative Behandlung der Retrodeviationen des Uterus.

Die relative Hiiufigkeit von Beckenverengerungen bei verschiedenen Raçen.

Die beste Methode der Bauchdeckennaht zur Vermeidung von Bauchbriichen.

Die Behandlung der Beckoneiterung $\cdot e n$.

Die Liste der Referenten ist noch nicht entgiiltig festgestellt. Der I. Con gress in Brüssel (1892) war von über ;Í00 T·eilwehmern besucht, überwiegend aus Frankreich und England. Es ist im Plane eine „Internationale Gynäkologische Gesellschaft" zu bilden, um dadurch die Einrichtung dieser Oongresse fester zu gründen.

Dr. Henry C. Coe (New-York, City 27, East, 64th Street) versendet folgende die Gaze-Drainage betreft'ende Umfrage an die Fachgenossen:

Benützen Sie die Gaze-Drainage nach Ausschabung des Uterus in septischen oder in nicht septischen Fallen, oder in beiden?

Bedienen Sie sich der G aze - Drainage in der Bauch - Chirurgie und in was für Fallen?

Wendeii Sie nur Gaze an oder diese zusammen mit einem Glas-Drain?

Sind Sie der Meinung, dass die Gaze septische Stoffe wegschaffe oder dass sie ledigl $\cdot$ ich rauhe Flachen und Krankhcitsheide bedecke, bis sie durch plastische Lympho abgekapselt sind? Gerade von deutscher Seite können auf diese Fragen sehr bestimmte Ant-worten gegeben werden. 
Personal-NachricMen.

Professor Kossel , unser verehrter Mitarbeiter, hat einen Ruf nach Marburg als Professor der Hygiene angenominon. Wir freuen uns dieser Anerkennung und begleiten unseri Freund mit den besten Wünschen.

Privatdocent Dr. F. Droysen in Göttingen ist zum ao. Professor ernannt worden. 1/1/8enso Dr. Fedoro $\Lambda \mathrm{V}$ in $\Lambda$ Yarschau.

Gíestorben Allessandro Cuzzi, Professor der Geburtshülfe und Gynä-kologie an der Universität Pavia, ein Schüler von Porro, in seiner Heircat besonders geschätzt wegen seiner Verdienste um das Hebammenwesen und auch in Deutschland bekannt und geachtet durch zahlreiche treffliehe Arbeiten. 